OPEN ACCESS

Edited by:

Ignacio Arechaga,

University of Cantabria, Spain

Reviewed by:

Yosuke Tashiro,

Shizuoka University, Japan

Paola Sperandeo,

University of Milan, Italy

*Correspondence:

Sophie Bleves

bleves@imm.cnrs.fr

Specialty section:

This article was submitted to Microbial Physiology and Metabolism, a section of the journal Frontiers in Microbiology

Received: 25 February 2019 Accepted: 15 May 2019 Published: 05 June 2019

Citation:

Berni B, Soscia C, Djermoun S, Ize B and Bleves S (2019) A Type VI

Secretion System Trans-Kingdom Effector Is Required for the Delivery of

a Novel Antibacterial Toxin in

Pseudomonas aeruginosa.

Front. Microbiol. 10:1218.

doi: 10.3389/fmicb.2019.01218

\section{A Type VI Secretion System Trans-Kingdom Effector Is Required for the Delivery of a Novel Antibacterial Toxin in Pseudomonas aeruginosa}

\author{
Benjamin Berni, Chantal Soscia, Sarah Djermoun, Bérengère Ize and Sophie Bleves* \\ LISM, IMM (Institut de Microbiologie de la Méditerranée), CNRS and Aix-Marseille Univ, Marseille, France
}

Pseudomonas aeruginosa has evolved multiple strategies to disarm and take advantage of its host. For this purpose, this opportunist pathogen has particularly developed protein secretion in the surrounding medium or injection into host cells. Among this, the type $\mathrm{VI}$ secretion system (T6SS) is utilized to deliver effectors into eukaryotic host as well as target bacteria. It assembles into a contractile bacteriophage tail-like structure that functions like a crossbow, injecting an arrow loaded with effectors into the target cell. The repertoire of T6SS antibacterial effectors of $P$. aeruginosa is remarkably broad to promote environmental adaptation and survival in various bacterial communities, and presumably in the eukaryotic host too. Here, we report the discovery of a novel pair of antibacterial effector and immunity of $P$. aeruginosa, Tle3 and Tli3. Tli3 neutralizes the toxicity of Tle3 in the periplasm to protect from fratricide intoxication. The characterization of the secretion mechanism of Tle3 indicates that it requires a cytoplasmic adaptor, Tla3, to be targeted and loaded onto the VgrG2b spike and thus delivered by the H2-T6SS machinery. Tla3 is different from the other adaptors discovered so far and defines a novel family among T6SS with a DUF2875. Interestingly, this led us to discover that VgrG2b that we previously characterized as an anti-eukaryotic effector possesses an antibacterial activity as well, as it is toxic towards Escherichia coli. Excitingly Tli3 can counteract VgrG2b toxicity. VgrG2b is thus a novel trans-kingdom effector targeting both bacteria and eukaryotes. VgrG2b represents an interesting target for fighting against $P$. aeruginosa in the environment and in the context of host infection.

Keywords: virulence, type VI secretion system, bacterial competition, VgrG, Tle, immunity, adaptor, chaperone

\section{INTRODUCTION}

Pseudomonas aeruginosa is one of the most virulent opportunistic pathogens, being responsible for various diseases such as acute infections of lungs and burned skin that can lead to septicemia more particularly in immunocompromized patients, or broncho-alveolar colonization in cystic fibrosis sufferers. P. aeruginosa has been classified in 2017 as critical by the WHO in the top 
three lists of antibiotic resistant bacteria (WHO, 2019). $P$. aeruginosa has developed various pathogenicity strategies among which protein secretion or protein delivery into target cells is key. Indeed, this pathogen possesses five of the six secretion systems so far identified among Gram-negative bacteria, if we exclude the T9SS (type IX secretion system) restricted to one phylum, and remarkably in several copies for most of them (Bleves et al., 2010).

The type VI secretion system (T6SS) was first discovered in the context of eukaryotic host infection (Mougous et al., 2006; Pukatzki et al., 2006) and later during bacterial competition (Hood et al., 2010), which seems to be its primary function (Cianfanelli et al., 2016b). The T6SS confers a fitness advantage (1) in environmental niches against rival bacteria (inter- and intraspecies competitiveness have been described) and (2) in the eukaryotic host towards commensal bacteria (Cianfanelli et al., 2016b). Indeed, recent studies have highlighted a novel role for T6SS-dependent antibacterial responses in interbacterial competition in the mammalian gut (Hecht et al., 2016; Sana et al., 2016b), suggesting that T6SSs may be important not only in shaping microbial community composition but also in governing interactions between the microbiota and invading pathogens. Interestingly, several T6SSs are also known to target both cell type genus such as the T6SS of Vibrio cholera (Pukatzki et al., 2006; MacIntyre et al., 2010) and P. aeruginosa (Sana et al., 2012) (Russell et al., 2013). Even more remarkably, three T6SS effectors of $P$. aeruginosa, namely, PldA (also called Tle5a), PldB (Tle5b), and TplE (Tle4) (Jiang et al., 2014, 2016) have been called "trans-kingdom effectors" since these toxins can target both prokaryotic and eukaryotic cells (Bleves, 2016). Indeed, toxins are usually directed against eukaryotic cells (like $\mathrm{AB}$ toxins or RTX pore-forming toxins) or against rival bacteria (like bacteriocins).

The T6SS functions as a dynamic contractile phage taillike structure anchored in the bacterial cell envelope that delivers effector proteins directly into the target cell in a one-step manner. T6SS includes a contractile sheath that cover a nanotube of stacked Hcp topped with a membranepuncturing spike made of VgrG and PAAR (proline-alaninealanine-arginine repeat) proteins (Basler, 2015). The sheath can contract and inject the arrow loaded with effectors into the target cell. Characterizing the repertoire of effectors delivered by the T6SS has highlighted a great diversity in terms of effector activities, host cell targets, and mode of recruitment by the T6SS machinery. In brief, there are two broad effector categories: the "specialized" effectors fused to components of the machinery (evolved VgrG, evolved PAAR, and evolved Hcp have been described so far) and the "cargo" effectors (Cianfanelli et al., 2016b). The later are addressed to the T6SS machinery by binding directly one of the arrow components (VgrG, PAAR, and Hcp) or by being targeted through cytoplasmic adaptor proteins also called chaperones. To date, three families of adaptor have been described, the first one harboring a DUF4123 (Miyata et al., 2013; Salomon et al., 2014; Liang et al., 2015; Unterweger et al., 2015), the second a DUF1795 (Alcoforado Diniz and Coulthurst, 2015; Whitney et al., 2015), and the third one a DUF2169 (Bondage et al., 2016). In line with this, many effector-encoding genes are found in close proximity to $\operatorname{vgr}, h c p$, and paar or adaptor genes. Finally to protect themselves from self-intoxication or from antibacterial toxins injected by neighboring sibling cells, bacteria always synthesize immunity proteins, which are encoded by adjacent genes (Benz and Meinhart, 2014).

$P$. aeruginosa encodes three distinct T6SS loci, H1- to H3-T6SS. While H1-T6SS has only been involved in antibacterial activity so far (Sana et al., 2016a; LaCourse et al., 2018), H2-T6SS and H3-T6SS can target both bacterial and eukaryotic cells possessing even as said earlier transkingdom effectors (Sana et al., 2012, 2015; Russell et al., 2013; Jiang et al., 2014, 2016; Burkinshaw et al., 2018). We discovered the anti-eukaryotic function of the H2-T6SS machinery that promotes the uptake of $P$ aeruginosa by non-phagocytic cells (Sana et al., 2012). The two phospholipases D mentioned earlier, PldA (Tle5a) and PldB (Tle5b), delivered, respectively, by $\mathrm{H} 2$-T6SS and $\mathrm{H} 3$-T6SS machineries participate in the host kinase pathway hijacking that facilitates further entry of $P$. aeruginosa (Jiang et al., 2014). The evolved VgrG2b effector (Sana et al., 2015) is delivered by H2-T6SS into epithelial cells where it targets the $\gamma$-tubulin ring complex, a microtubule-nucleating multiprotein complex to promote a microtubule-dependent internalization of $P$. aeruginosa. Finally TplE (Tle4), which is secreted by the H2-T6SS machinery, promotes autophagy in epithelial cells once localized to the endoplasmic reticulum (Jiang et al., 2016). Interestingly, PldA (Tle5a), PldB (Tle5b), and TplE (Tle4) have also been identified as antibacterial phospholipases of the Tle (type VI lipase effectors) family (Russell et al., 2013). They work by affecting membrane integrity of the rival bacteria (Russell et al., 2013; Jiang et al., 2014, 2016). More precisely, PldA degrades the major constituent of bacterial membranes, the phosphatidylethanolamine (Russell et al., 2013).

In the present study, we have discovered a novel antibacterial toxin, Tle3, and its cognate immunity, Tli3, whose genes are encoded downstream of $v r g G 2 b$. By characterizing the secretion mechanism of Tle3 by H2-T6SS, we showed that it requires Tla3, a cytoplasmic adaptor of a unique family, to be targeted to the VgrG2b spike. Interestingly, we also found that the C-terminal extension of $\mathrm{VgrG} 2 \mathrm{~b}$ is toxic towards Escherichia coli making VgrG2b a new trans-kingdom effector of $P$. aeruginosa.

\section{MATERIALS AND METHODS}

\section{Bacterial Strains, Growth Conditions, and Plasmid Construction}

All $P$. aeruginosa and $E$. coli strains used in this study are described in Supplementary Table S1. Briefly, the E. coli K-12 $\mathrm{DH} 5 \alpha$ and CC118 $\lambda$ Pir were used for cloning procedures. The BL21(DE3)pLysS and BTH101 were used for protein production and BACTH analyses, respectively. Strains were grown in LB or in TSB medium (for P. aeruginosa) at 37 or $30^{\circ} \mathrm{C}$. Specific growth conditions are specified in the text when necessary. Recombinant plasmids were introduced into $P$. aeruginosa by triparental mating 
using the conjugative properties of the helper plasmid pRK2013 (Supplementary Table S1). Plasmids were maintained by the addition of ampicillin (50 $\mu \mathrm{g} / \mathrm{ml})$, kanamycin $(50 \mu \mathrm{g} / \mathrm{ml})$, chloramphenicol $(30 \mu \mathrm{g} / \mathrm{ml})$, streptomycin $(30 \mu \mathrm{g} / \mathrm{ml}$ for E. coli and $2,000 \mu \mathrm{g} / \mathrm{ml}$ for $P$. aeruginosa), or gentamicin $(30 \mu \mathrm{g} / \mathrm{ml}$ for E. coli and $115 \mu \mathrm{g} / \mathrm{ml}$ for $P$. aeruginosa). Expression of genes from pT7 in BL21(DE3)pLysS was blocked with $0.4 \%$ of glucose and induced in exponential phase $\left(\mathrm{OD}_{600}=0.4-0.6\right)$ for $3 \mathrm{~h}$ with $1 \mathrm{mM}$ of IPTG. Cloning procedures were described in Sana et al. (2015). The plasmids used and constructed are described in Supplementary Table S1, the list of oligonucleotides (synthesized by Eurogentec or IDT) is given in Supplementary Table S2.

\section{Cloning Procedures for $P$. aeruginosa Mutants}

To generate $P$. aeruginosa mutants, 500 bp upstream and 500 bp downstream of the gene to be deleted were amplified by overlapping PCR with Q5 high fidelity DNA polymerase (NEB) using primers listed in Supplementary Table S1. The PCR product was cloned in pKNG101 suicide vector by one-step sequence and ligation-independent cloning (SLIC) (Jeong et al., 2012), which was then sequenced. pKNG101 derivatives,

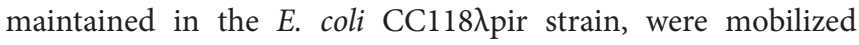
in $P$. aeruginosa strains. The mutants, in which the double recombination events occurred, were confirmed by PCR analysis.

\section{Heterologous Toxicity Assays}

E. coli BL21(DE3)pLysS containing plasmids producing cytoplasmic or periplasmic targeted proteins were grown overnight at $37^{\circ} \mathrm{C}$ in $\mathrm{LB}$ with $0.4 \%$ of glucose. About $10 \mu \mathrm{l}$ drops of bacterial suspensions serially diluted were spotted onto LB agar plates containing $0.1 \mathrm{mM}$ IPTG or $0.4 \%$ glucose and cells were grown for $16 \mathrm{~h}$ at $37^{\circ} \mathrm{C}$.

\section{Bacterial Two-Hybrid Assay}

Protein-protein interactions were assessed with the adenylate cyclase-based two-hybrid technique using protocols published previously (Karimova et al., 1998; Battesti and Bouveret, 2012). Briefly, the proteins to be tested were fused to the isolated T18 and T25 catalytic domains of the Bordetella adenylate cyclase. After introduction of the two plasmids producing the fusion proteins into the reporter BTH101 strain, plates were incubated at $30^{\circ} \mathrm{C}$ for $24 \mathrm{~h}$. Three independent colonies for each transformation were inoculated into $600 \mu \mathrm{l}$ of LB medium supplemented with ampicillin $(50 \mu \mathrm{g} / \mathrm{ml})$, kanamycin $(50 \mu \mathrm{g} / \mathrm{ml})$, and IPTG $(0.5 \mathrm{mM})$. After overnight growth at $30^{\circ} \mathrm{C}, 5 \mu \mathrm{l}$ of each culture was spotted onto LB agar plates supplemented with ampicillin, kanamycin, IPTG, and 5-bromo-4-chloro3-indonyl-D-galactopyrannoside (X-gal, $40 \mu \mathrm{g} / \mathrm{ml}$ ) and incubated for $16 \mathrm{~h}$ at $30^{\circ} \mathrm{C}$.

\section{Protein Purification by Affinity Chromatography}

E. coli BL21(DE3)plysS cells carrying the pRSFDUET-1 and pETDUET-1 derivatives were grown at $37^{\circ} \mathrm{C}$ in $\mathrm{LB}$ to an
$\mathrm{OD}_{600} \sim 0.5$ and the expression of the PA0262, PA0261, PA0260, or PA0259 genes was induced with IPTG $(1 \mathrm{mM})$ for $3 \mathrm{~h}$ at $37^{\circ} \mathrm{C}$. Cells were harvested by centrifugation at $1,914 \times g$ for $30 \mathrm{~min}$ at $4^{\circ} \mathrm{C}$. The cell pellet was resuspended in Tris- $\mathrm{HCl}$ $50 \mathrm{mM} \mathrm{pH}$ 8.0, NaCl $150 \mathrm{mM}$, Triton X-100 0.1\%, lysozyme $0.5 \mathrm{mg} / \mathrm{ml}$, and EDTA $1 \mathrm{mM}$ and stored at $-80^{\circ} \mathrm{C}$. Cells were supplemented with DNase $(20 \mu \mathrm{g} / \mathrm{ml}), \mathrm{MgCl}_{2}$, and phenylmethylsulfonyl fluoride $1 \mathrm{mM}$ and cells were lysed by three passages at the Emulsiflex-C5 (Avestin), and lysates were clarified by centrifugation at $16,000 \times g$ for $30 \mathrm{~min}$. The supernatant was loaded onto a 5-ml StrepTrap HP (GE Healthcare) column and then washed with $50 \mathrm{mM}$ Tris- $\mathrm{HCl}$ $\mathrm{pH} 8.0,150 \mathrm{mM} \mathrm{NaCl}$ at $4^{\circ} \mathrm{C}$. The fusion protein was eluted in the affinity buffer supplemented with $2.5 \mathrm{mM}$ desthiobiotin. Peak fractions were pooled and loaded onto a Superose 200 $10 / 300$ column (GE Healthcare) equilibrated in $50 \mathrm{mM}$ Tris- $\mathrm{HCl}$ pH 8.0, $50 \mathrm{mM} \mathrm{NaCl}$.

\section{Fractionation of $P$ aeruginosa}

Fractionation of cells into spheroplasts (cytoplasm and membranes) and periplasmic fractions were done as previously described (Ize et al., 2014). Proteins corresponding to the cytoplasm and periplasm fractions or to insoluble material were resuspended in loading buffer.

\section{Protein Secretion}

$P$. aeruginosa strains were grown at $25^{\circ} \mathrm{C}$ in TBS for $24 \mathrm{~h}$. Cells corresponding to $10 \mathrm{U} \mathrm{DO}_{600}$ and extracellular medium were separated by centrifugation at $2,000 \times g$ for $10 \mathrm{~min}$ at room temperature. $2 / 3$ of the supernatants were collected and centrifuged at $13,000 \times g$ for $5 \mathrm{~min}$ at room temperature. Proteins contained in the supernatant were precipitated with tricholoro-acetic acid (TCA, 15\%) for $3 \mathrm{~h}$ at $4^{\circ} \mathrm{C}$. Samples were centrifuged at $13,000 \times g$ for $30 \mathrm{~min}$ at $4^{\circ} \mathrm{C}$, pellets washed with $90 \%$ acetone, and resuspended in loading buffer.

\section{SDS-PAGE and Western-Blot}

Protein samples derived from equivalent amounts of culture (i.e., optical density equivalents) resuspended in loading buffer were boiled and separated by SDS-PAGE. Proteins were then stained by Coomassie-blue or immunodetected as described before (Sana et al., 2015) using primary polyclonal antibodies directed against His6 epitope-tag (Penta His, Qiagen, dilution 1:1,000), V5 epitope-tag (Bethyl Laboratories, dilution 1:1,000), Strep epitope-tag (IBA StrepMAB Classic, dilution 1:000), DsbA (kindly gifted by K. E. Jaeger - university of Heinrich-Heine, dilution 1:25,000), or monoclonal antibodies directed against EF-Tu (Hycult-biotech, dilution 1:20,000), XcpY (laboratory collection, dilution 1:5,000), and TolB (laboratory collection, dilution 1:500). Peroxidaseconjugated anti-Mouse or anti-Rabbit IgGs (Sigma, dilution 1:5,000) were used as secondary antibodies. Nitrocellulose membranes were revealed with homemade enhanced chemiluminescence and were scanned using ImageQuant LAS4000 analysis software (GE Healthcare Life sciences).

Protein samples equivalent to $0.1 \mathrm{OD}_{600}$ units were loaded for whole cell and spheroplasts analysis while protein samples 
equivalent to $0.2 \mathrm{OD}_{600}$ units were used for cytoplasm or periplasm analysis and protein samples equivalent to $1 \mathrm{OD}_{600}$ units were used for extracellular medium analysis.

\section{Bacterial Competition Assays}

Intraspecific competition assays between $P$. aeruginosa strains were performed as previously described (Jiang et al., 2014) with modifications. The prey cells carry pJN105 vector $\left(\mathrm{Gm}^{\mathrm{R}}\right)$ to allow counterselection. Overnight cultures of $P$. aeruginosa attacker and prey cells were mixed in a 10:1 (attacker:prey) ratio and harvested by centrifugation at $3,724 \times g$ for $5 \mathrm{~min}$. The pellet was resuspended in $200 \mu \mathrm{l}$ of PBS $1 \mathrm{X}$ and spotted onto $0.45-\mu \mathrm{m}$ nitrocellulose membranes overlaid on a $1 \%$ bactoagar plate. After $24 \mathrm{~h}$ of incubation at $37^{\circ} \mathrm{C}$, cells were resuspended in $2 \mathrm{ml}$ of $\mathrm{PBS} 1 \mathrm{X}$, normalized to an $\mathrm{OD}_{600 \mathrm{~nm}}$ of 0.5 , and $10 \mu \mathrm{l}$ of bacterial serially diluted $\left(10^{-1}\right.$ to $\left.10^{-6}\right)$ were spotted onto selective LB agar plates containing gentamicin $(125 \mu \mathrm{g} / \mathrm{ml})$. Significant growth difference of the prey bacteria for each competition assay was computed by one-way ANOVA (Stat Plus) and unpaired Student's Test (Excel).

\section{RESULTS}

\section{Tle3 Is a Novel Antibacterial Toxin of $P$. aeruginosa}

The analysis of the $\operatorname{vgrG} 2 b$ genetic environment revealed the presence of the PA0260 gene encoding a protein with a $\alpha / \beta$ hydrolase domain (PF00561) and a putative Ser-Asp-His catalytic triad used by various esterase enzymes and has thus been classified in the Tle3 family of antibacterial Tle toxins (Figure 1A; Russell et al., 2013). The immunities of Tle proteins, which are lipolytic toxins active in the periplasm of the prey bacterium, are localized in or exposed to the periplasm where they neutralize the cognate toxin (Russell et al., 2013; Jiang et al., 2014, 2016; Flaugnatti et al., 2016). The two genes surrounding tle3, PA0259 and PA0261, are good candidates as Tle3 immunity. Indeed, sequence comparisons of PA0259 and PA0261 showed that PA0261 is homologous to Tsi6, the immunity protein of a H1-T6SS effector called Tse6, and PA0259 to TplEi (Tli4), the immunity of TlpE (Tle4), a H2-T6SS effector (Lu et al., 2014; Jiang et al., 2016). We used the SignalP 4.1 server (Nielsen, 2017) to predict the cellular localization of the two immunity candidates. While a Sec signal sequence is predicted at the $\mathrm{N}$-terminal extremity of PA0261, the analysis of the PA0259 sequence did not reveal any. However, three upstream ATG can be found in frame with the annotated ATG of PA0259 (Supplementary Figure S1). The sequence of the proteins synthesized from the two first ATG $\left(\mathrm{ATG}_{1}\right.$ and $\mathrm{ATG}_{2}$ ) presents then a $\mathrm{N}$-terminal signal peptide, while the protein synthesized from the last codon $\left(\mathrm{ATG}_{3}\right.$ ) does not. Moreover, a ribosome-binding sequence (RBS) can be found only upstream of $\mathrm{ATG}_{1}$ and with a significant Kolaskar score that indicates a strong probability to be used as an initiation codon (Kolaskar and Reddy, 1985). Altogether, these data tend to indicate an incorrect annotation of the start codon of PA0259 and that ATG should $_{1}$ be considered for the initiation of PA0259 translation. Such a correction has been already seen for Tli5 (PA3488) the immunity protein of PldA (Tle5a) of P. aeruginosa (Russell et al., 2013). In conclusion of this in silico analysis, the two putative immunities may harbor a Sec signal peptide that suggests a periplasmic localization in agreement with Tle3 activity in this compartment.

To demonstrate the antibacterial activity of Tle3 and to identify its immunity protein, we developed a heterologous toxicity assay in E. coli on the basis that Tle3 should be toxic when produced in the periplasm of $E$. coli and that it should be counteracted by the co-production of its immunity protein. In order to artificially address Tle3 to the periplasm of $E$. coli the tle 3 sequence has been cloned in frame with the sequence coding the PelB signal peptide on the $\mathrm{pET} 22 \mathrm{~b}$ vector under a $\mathrm{P}_{\mathrm{T} 7}$ promoter. PA0259 from $\mathrm{ATG}_{1}$ and PA0261 were respectively cloned in pRSF-DUET, a higher copy-number vector, to allow a maximal co-expression with ss-tle 3 in the E. coli BL21(DE3) pLysS strain. The correct production and localization of all the recombinant proteins in E. coli has been verified by western blot after cell fractionation (Supplementary Figure S2). The results presented in Figure 1B indicate that whereas the cytoplasmic production of Tle3 was not toxic, Tle 3 targeting to the periplasm led to E. coli killing. Moreover, while PA0259 had no effect, the coproduction of PA0261 in the periplasm protected the cells against the toxicity of Tle3. We verified that the sole overproduction of PA0259 and PA0261 was not toxic to E. coli. As PA0261 neutralized Tle3 toxicity, we called it "Tli3" for Type VI lipase immunity (Figure 1A). During this study, we observed that the protection conferred by Tli3 coproduction could be sometimes partial and we solved this issue by cloning in tandem tli3 and tle 3 on the same plasmid like they are organized on $P$. aeruginosa genome (Figure 1B, line 8). The importance of this genetic link is the proof of the close connection between these two proteins as a pair of toxin-antitoxin.

\section{Tli3 (PA0261) Is the Immunity Protein of Tle3}

As the immunities bind specifically their effector, which is suggested by the release of Tle3 toxicity by Tli3, we tested the physical interaction between both proteins by co-purification with affinity chromatography (Figure 2A and Supplementary Figure S6). A cytoplasmic Strep-tagged version of Tli3 was engineered by fusing the tag to the mature domain of Tli3, lacking its signal peptide. The recombinant protein was coproduced in E. coli BL21(DE3) pLysS with the cytoplasmic His ${ }_{10}$-tagged Tle3. The bacterial lysate was loaded to a StrepTactin matrix (see section "Materials and Methods"), and Tli3 ${ }_{\text {Strep }}$ was eluted with desthiobiotin. The presence of Tle $3_{\text {His }}^{\mathrm{C}}$ was controlled in the elution fraction with anti-His antibodies. As showed in Figure 2A and Supplementary Figure S6, Tle ${ }^{\mathrm{C}}{ }_{\text {His }}$ was found in the eluted fraction only upon coproduction with $\mathrm{Tli}^{\mathrm{C}}{ }_{\text {Strep }}$ (left panel). Indeed when produced alone in E. coli, Tle $3{ }^{\mathrm{C}}$ His was not purified by affinity chromatography (right panel). As expected for an immunity protein, Tli3 directly interacts with Tle3. 

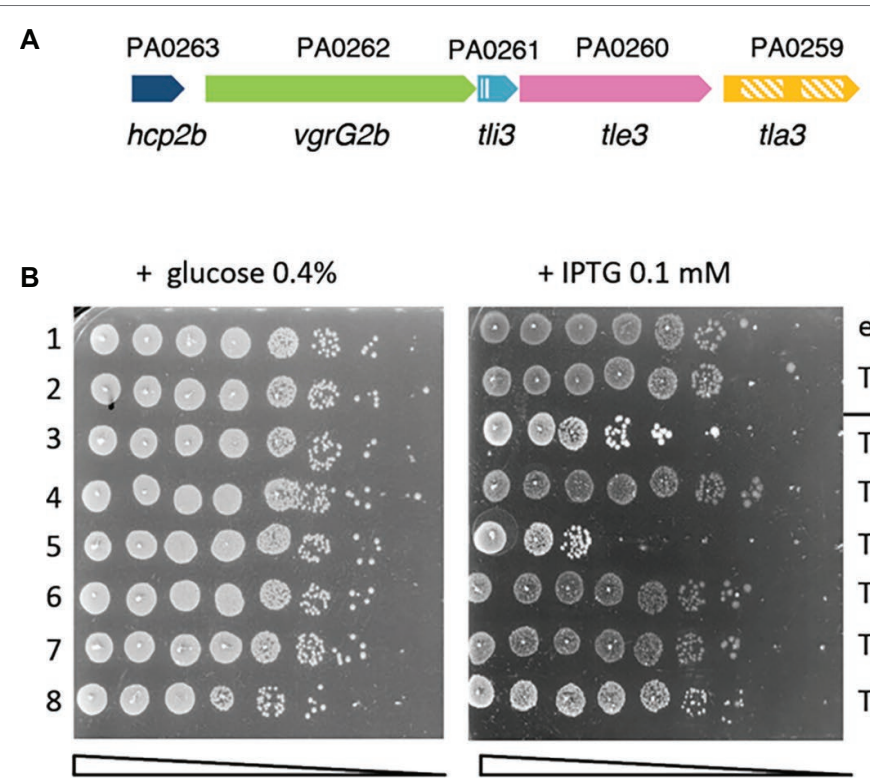

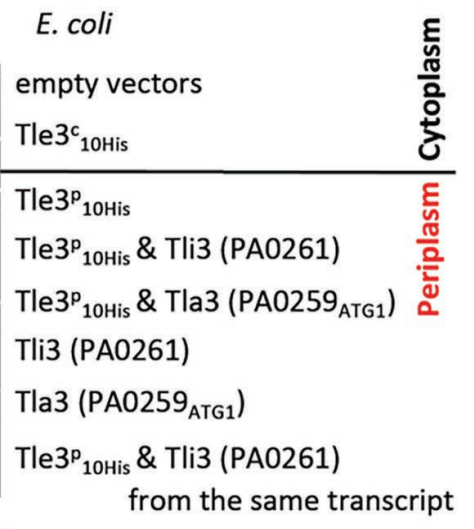

FIGURE 1 | (A) vgrG2b island organization. The genes are labeled with the given name (i.e., hcp2b) and are indicated by their annotation number (e.g., PA0263). The Sec signal peptide of Tli3 (PA0261) and the two DUF2875 of Tla3 (PA0259) are represented with stripped boxes. (B) The Tle3 periplasmic toxicity is counteracted by Tli3 (PA0261). Serial dilutions (from non-diluted to $10^{-7}$ ) of normalized cultures of E. coli BL21(DE3)pLysS producing the wild-type Tle3 in the cytoplasm, called Tle3 (from pVT1, a pETDuet-1 derivative) or in the periplasm, called Tle3 ${ }^{p}$ [from pSBC81, a pET22b(+) derivative yielding a fusion of Tle3 with a Sec signal peptide] were spotted on LB agar plates supplemented (left panel) with $0.4 \%$ glucose or (right panel) with $0.1 \mathrm{mM} \mathrm{IPTG.} \mathrm{Glucose} \mathrm{and} \mathrm{IPTG} \mathrm{allow}$ respectively repression and induction of the gene encoding the T7 RNA polymerase. When indicated Tli3 (PA0261) or Tla3 (PA0259) were produced in the periplasm from pVT8, pSBC107 and pVT9, respectively, pRSFDuet-1 derivatives. Line 1: pET22b(+) and pRSFDuet-1, line 2: pVT1 and pRSFDuet-1, line 3: pSBC81 and pRSFDuet-1, line 4: pSBC81 and pVT8, line 5: pSBC81 and pVT9, line 6: pVT8 and pET22b(+), line 7: pVT9 and pET22b(+), and line 8: pSBC107 is a pRSFDuet-1 derivative producing Tli3 (PA0261) and Tle $3^{\circ}$ from the same transcript.

To go further into Tli3 characterization, we chose to determine its cellular localization in $P$. aeruginosa. All the immunity proteins identified so far for Tle proteins are localized in the periplasm or associated to the periplasmic side of the outer membrane (Russell et al., 2013; Jiang et al., 2014, 2016; Flaugnatti et al., 2016) in order to counteract their cognate toxin. A chromosomally encoded $\mathrm{Tli}_{\mathrm{V} 5}$ translational fusion was engineered in order to specifically immunodetect the protein in $P$. aeruginosa (Supplementary Table S1). After fractionation of $P$. aeruginosa (Figure 2B), Tli3 was readily observed in the same fraction as DsbA that catalyzes intrachain disulfide bond formation as peptides emerge into the periplasm. This indicates a periplasmic localization for Tli3 in P. aeruginosa in agreement with the presence of a Sec signal peptide and our working hypothesis suggesting an immunity function.

\section{Tle3 Protein-Protein Interaction Network}

To further characterize Tle3, we performed a bacterial two-hybrid (BACTH) assay with the other gene products of the $\operatorname{vgrG} 2 b$ operon hypothesizing that a genetic link could reflect proteinprotein interaction. The sequences coding PA0259 and Tli3 (PA0261) after their signal sequences and Tle3 were cloned downstream and upstream the T18 or T25 domains of the Bordetella adenylate cyclase. Because of the high molecular weight of VgrG2b and since the interaction of another Tle with a VgrG in entero-aggregative E. coli (EAEC) was previously delimitated to the C-terminal domain of $\mathrm{VgrG}$
(Flaugnatti et al., 2016), we cloned the sequences encoding the C-terminal extension (domains 1, 2, and 3) and a truncated version harboring only the DUF2345 and the transthyretin-like (TTR) domains (domains 1 and 2) of VgrG2b downstream the T18 and T25 domains (Figure 3A).

Unexpectedly, the sole interaction revealed by the BACTH assay for Tle3 was with PA0259 (Figure 3B) since only the T18/T25-Tle3 and T18/T25-PA0259 fusion proteins coproduction activated the expression of the reporter gene. This assay did not confirm the interaction between Tle 3 and Tli3 observed by copurification and did not evidence an interaction with VgrG2b. Interestingly, Tle3 and PA0259 did not interact anymore when they were fused upstream the T18 and T25 domains suggesting that both proteins interact via their C-terminal domains (Supplementary Figure S3A). To go further in characterizing the interaction between Tle3 and PA0259, we constructed truncated variants taking into account their domain organization (Figure 3A). We further delimitated the interacting domain of Tle 3 to its extreme C-terminus (the DUF3274 domain) since only the truncated T18-Tle3D2 construct still interacts with T25-PA0259 (Figure 3C). We did not find the domain of interaction within PA0259 as none of the DUF2875 domains alone interacts with Tle 3 or this may suggest that both of them are required for the interaction (Figure 3C).

Next, we took advantage of all the constructs we made to test other interactions. The BACTH assay also showed that 

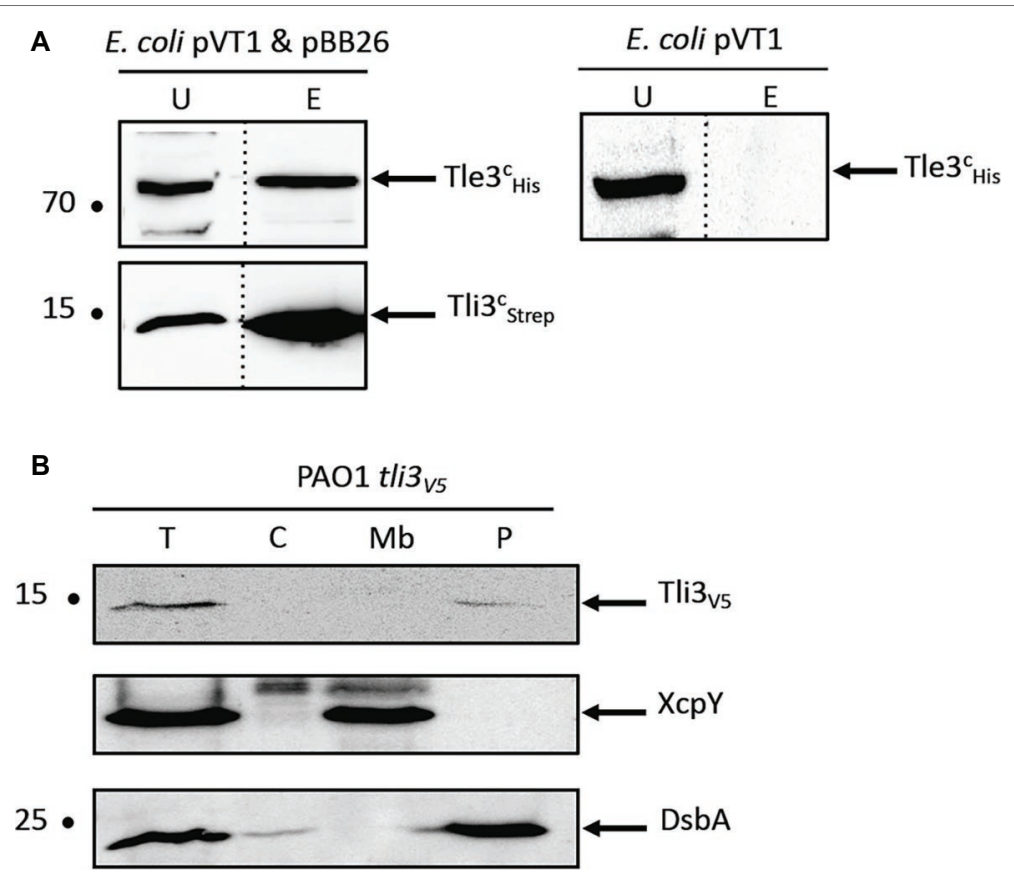

FIGURE 2 | (A) Tli3 (PA0261) interacts with Tle3. Copurification assay on StrepTactin column of Tli3c Strep $_{\text {With Tle3c10 His }}$ produced in E. coli BL21(DE3)pLysS produced from pBB26 and pVT1 respectively. The unbound (U) and eluted (E) fractions were collected and subjected to SDS-PAGE (10.5\%) and Western blot analyses using anti-His antibody (Upper) and anti-streptavidin antibody (Lower). The position of the proteins and the molecular mass markers (in kDa) are indicated. (B) Tli3 (PA0261) is a periplasmic protein in $P$. aeruginosa. Cells of $P$. aeruginosa PAO1 tli3 $\mathrm{V}_{55}$ were subjected to fractionation and immunoblotting using antibodies directed against the V5 tag, XcpY and DsbA. XcpY and DsbA were used as membrane and periplasmic controls, respectively. T, whole cell; C, cytoplasm; Mb, total membrane; P, periplasm. The position of the proteins and the molecular mass markers (in $\mathrm{kDa}$ ) are indicated.

PA0259 interacts with both forms of the VgrG2b C-terminal extension (Figure 3B). The domain of interaction on VgrG2b is thus at least constituted by the DUF2345 and TTR domains. We also observed that PA0259 at least dimerizes since all the PA0259 constructs interact with each other whatever the orientation of PA0259 (Figures 3B,D; Supplementary Figure S3B). VgrG2b and Tli3 (PA0261) are also able to homomultimerize since all the constructs interact with each other (Figure 3D; Supplementary Figure S3B).

Taking into account the interactions revealed by the BACTH assay, we propose that the Tle3 toxin can be addressed to the H2-T6SS machinery VgrG2b component via PA0259. We thus named PA0259 “Tla3" for Type VI lipase adaptor protein.

\section{Tla3 (PA0259) Characterization}

To gain insight into the role of Tla3 during Tle 3 secretion, we first validated the interactions of Tla3 with Tle3 and VgrG2b by a complementary approach of co-purification by affinity chromatography. Two different tagged versions of Tla3 were engineered by fusing a Strep-tag or a 10Histag to the mature domain of Tla3 this leading to cytoplasmic tagged Tla3 proteins. The recombinant $\mathrm{Tla}^{\mathrm{C}}{ }_{\text {strep }}$ was coproduced in E. coli BL21(DE3) pLysS with Tle $3{ }_{\text {His }}^{\mathrm{C}}$, and the Tla $3{ }_{\text {His }}$ with 3 recombinant forms of Strep-tagged VgrG $2 b$ consisting in the full-length VgrG2b, or VgrG2b truncated for the extreme C-terminus (deletion of domain 3 in Figure 3A) or VgrG2b truncated for the extreme C-terminus and the TTR domain (deletion of domains 2 and 3 in Figure 3A). We initially tried with His-tagged VgrG2b but a problem of protein instability led us to shift for Strep-tagged VgrG2b. The bacterial lysates were loaded on a StrepTactin matrix, and $\mathrm{Tla} 3_{\text {Strep }}^{\mathrm{C}}$ or the three recombinant $\operatorname{VgrG} 2 \mathrm{~b}_{\text {Strep }}$ were eluted with desthiobiotin. The presence of Tle $3^{\mathrm{C}}{ }_{\text {His }}$ and of $\mathrm{Tla3}_{\text {His }} \mathrm{C}_{\text {was }}$ visualized in the elution fractions with anti-His antibodies. As shown in Figures 4A, 2A right panel and Supplementary Figure S6, Tle ${ }^{\mathrm{C}}$ His was only found in the eluted fraction upon coproduction with $\mathrm{Tla}^{\mathrm{C}}{ }_{\text {Strep. }}$. We observed that $\mathrm{Tla}^{\mathrm{C}}{ }_{\text {His }}$ is copurified only upon coproduction with the full length VgrG2b (Figure 4B and Supplementary Figure S6) or the VgrG2b truncated for the extreme C-terminus (Figure 4C and Supplementary Figure S6). Indeed when produced alone (Figure 4B and Supplementary Figure S6) or with VgrG2b truncated for the extreme C-terminus and the TTR domain (Figure 4D and Supplementary Figure S6), $\mathrm{Tla}^{\mathrm{C}}{ }_{\text {His }}$ was not purified by affinity chromatography. Since VgrG2b truncated for the extreme C-terminus still copurified with Tla3 we can exclude that this domain is required for the interaction. This is in line with the BACTH assay that showed an interaction between Tla3 and a truncated VgrG2b consisting in only the DUF2345 and TTR domains (domains 1 and 2, Figure 3A). Moreover, the deletion of the TTR domain affecting the copurification, one can conclude that this domain is key for the interaction. 

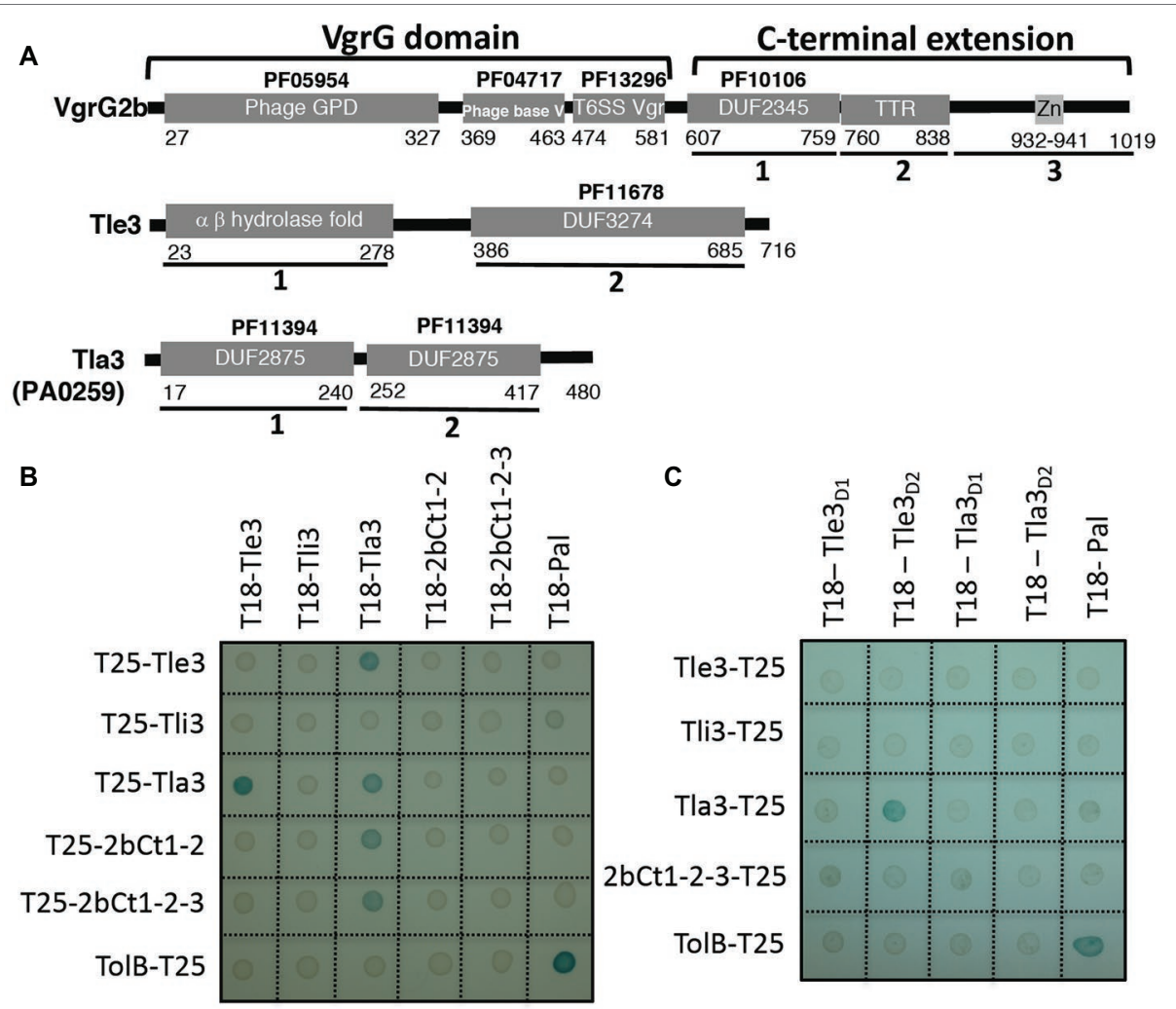

C

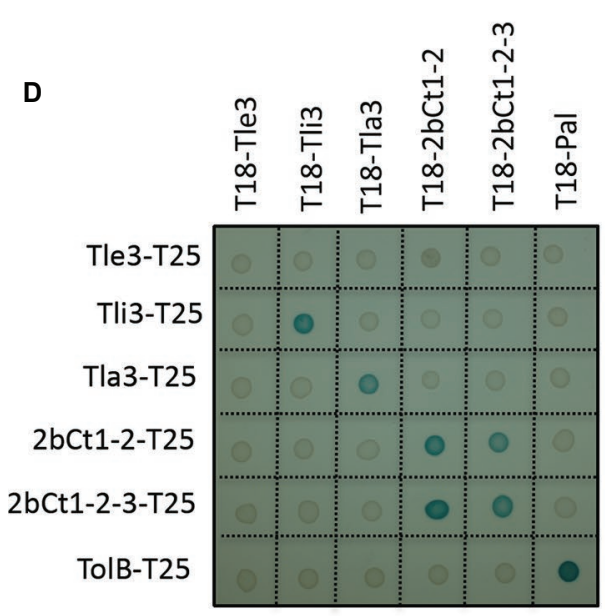

FIGURE 3 | (A) Tla3 (PA0259) interacts with the Tle3 toxin and with VgrG2b. Domain organization of VgrG2b, Tle3, and Tla3 (PA0259). The first 581 residues of VgrG2b carry the VgrG domain homologous to gp27 and gp5 phage tail proteins and consisting of three sub-domains. This is followed by the C-terminal extension, composed of a conserved domain of uncharacterized proteins (DUF2345, PF10106) (2bCt1), a TTR (transthyretin-like region) (2bCt2), and a putative zinc-dependent metallopeptidase pattern (LFIHEMTHVW signature, PS00142) (2bCt3). Tle3 architecture consists of an $\alpha, \beta$ hydrolase fold domain (Tle $3_{D 1}$ ) followed by a DUF3274 (Tle $3_{\mathrm{D} 2}$ ), Tla3 of a tandem of DUF2875 (Tla $3_{\mathrm{D} 1}$ and Tla3 $\mathrm{B}_{\mathrm{D} 2}$ ). (B-D) Bacterial two-hybrid assay. BTH101 reporter cells producing the indicated proteins or domains fused to the T18 or T25 domain of the Bordetella adenylate cyclase were spotted on X-gal indicator plates. The blue color of the colony reflects the interaction between the two proteins. ToIB and Pal are two proteins known to interact but unrelated to the T6SS. The experiment was performed in triplicate and a representative result is shown.

Taken together these data confirmed a direct interaction of Tla3 with Tle 3 on one side and with VgrG2b on the other side. By taking into account the BACTH data and the copurification with two truncated forms of $\operatorname{VgrG} 2 \mathrm{~b}$, the domain of interaction of VgrG2b with Tla3 can be delimitated to the TTR domain.
We then analyzed the cellular localization of Tla3 in $P$. aeruginosa that, according to its interactions with Tle3 and VgrG2b, should be cytoplasmic. As for Tli3, we engineered a chromosomally encoded Tla $3_{\mathrm{v} 5}$ translational fusion (Supplementary Table S1). Tla3 was indeed immunodetected in the cytoplasmic fraction (Figure 5A). One could note that 


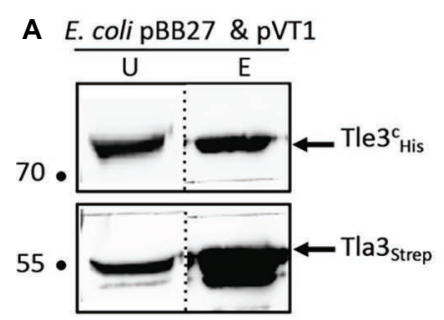

C

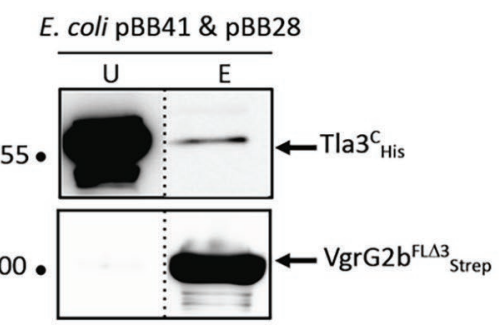

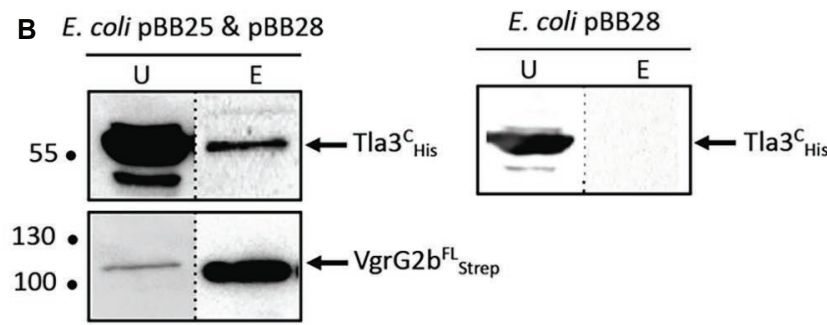

D

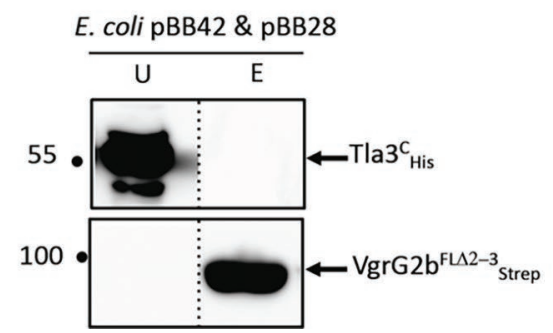

FIGURE 4 | Tla3 interaction with Tle3 (A) and VgrG2b (B-D). Copurification assay on StrepTactin column of Tla3 ${ }^{c}$ STREP with Tle3 $^{c}$ His $_{\text {is }}$ produced in E. coli BL21(DE3)

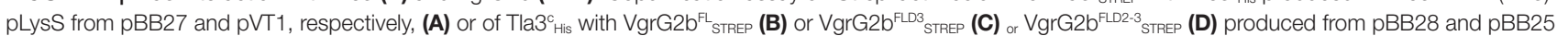
(B) or pBB41(C) or pBB42 (D). The unbound (U) and eluted (E) fractions were collected and subjected to SDS-PAGE (10.5\%) and Western blot analysis using anti-His antibody (upper) and anti-streptavidin antibody (lower). The dashed line separates lanes from non-adjacent part of the same gel. The position of the proteins and the molecular mass markers (in $\mathrm{kDa}$ ) are indicated.

in contrast with our first hypothesis suggesting an incorrect start codon for tla3 and our observation of the recombinant protein in the periplasm of E. coli (Supplementary Figure S1), Tla3 was totally absent from the periplasmic fraction of $P$. aeruginosa. To strengthen this result, each putative ATG was individually mutated on the chromosome of the PAO1 strain encoding the $\mathrm{Tla}_{\mathrm{V} 5}$ translational fusion (Figure 5B). In agreement with its cytoplasmic localization in $P$. aeruginosa, Tla3 was only produced if the fourth ATG was intact. Accordingly the Tla3 protein synthesized from this ATG is not predicted to possess a N-terminal signal peptide (Supplementary Figure S1). In conclusion, Tla3 is a cytoplasmic protein synthesized from the annotated translation $\operatorname{start}^{1}$ and this localization is in agreement with a role in the targeting of the toxin to the secretion machinery.

Next, we asked whether Tla3 is specific for Tle3 or if it can be required for the secretion of other substrates of the H2-T6SS machinery. Since Hcp secretion is the hallmark of a functional secretion system, we studied the secretion of Hcp2b whose gene is upstream $\operatorname{vgrG} b$ (Figure 1A). Like Allsopp et al. (2017), we deleted the rsmA gene to enable Hcp2b production and thus secretion by a PAO1 strain encoding a Hcp $2 b_{\text {His }}$ translational fusion (Figure 5C, compare lines 1 and 2). RsmA is a posttranscriptional regulator known to repress all three T6SS clusters of $P$. aeruginosa (Allsopp et al., 2017). This results in a massive secretion since $\mathrm{Hcp} 2 \mathrm{~b}_{\mathrm{His}}$ can be observed in the extracellular protein samples by Coomassie-blue staining (Figure 5C, lower panel). While Hcp $2 \mathrm{~b}_{\mathrm{His}}$ secretion was abolished

${ }^{1}$ pseudomonas.com in a $r s m A \operatorname{clp} V 2$ mutant, a H2-T6SS mutant, we observed that $\mathrm{Hcp} 2 \mathrm{~b}_{\mathrm{His}}$ is still secreted in the absence of Tla3 (Figure 5C, compare line 3 with line 4), suggesting that Tla3 is specific for the secretion of Tle3 but not for other H2-T6SS proteins. In line with this specific adaptor-toxin pair, $\mathrm{Tla}^{\mathrm{C}^{\mathrm{C}}}{ }_{\text {Strep }}$ did not copurify TplE (Tle4), another antibacterial phospholipase delivered by the H2-T6SS machinery (Figure 5D and Supplementary Figure S6).

Finally as the interaction with a VgrG can suggest that Tla3 is itself a T6SS effector, we studied whether Tla3 is secreted by $P$. aeruginosa. To this end, the $\operatorname{rsm} A$ gene was deleted from the PAO1 strain encoding a $\mathrm{Tla}_{\mathrm{V} 5}$ translational fusion. Whereas $\mathrm{Tla}_{\mathrm{V} 5}$ was better produced upon $\operatorname{rsmA}$ deletion (Figure 5E), this did not lead to immunodetection of $\mathrm{Tla}_{\mathrm{V} 5}$ in the extracellular medium although Hcp2b was readily observed by Coomassie-blue staining of the same samples. Tla3 is thus not an effector per se.

In conclusion, the cytoplasmic localization of Tla3 in $P$. aeruginosa is appropriate with a recruiting role of Tle 3 to the H2-T6SS machinery through an interaction with the TTR domain of VgrG2b. Tla3 is not co-secreted with Tle3. Tla3 role seems specific to Tle3 since it is not required for a functional H2-T6SS machinery and does not interact with TplE (Tle4), another H2-T6SS effector.

\section{Tle3 Secretion Mechanism by the H2-T6SS Machinery of $\boldsymbol{P}$ aeruginosa}

In order to study the antibacterial role and the secretion of Tle3 by $P$. aeruginosa, we performed intra-species bacterial competition assays between $P$. aeruginosa strains. This consists in studying the survival of prey bacteria lacking the tli3 immunity gene (by CFU counting of antibiotic resistant bacteria) co-cultivated 


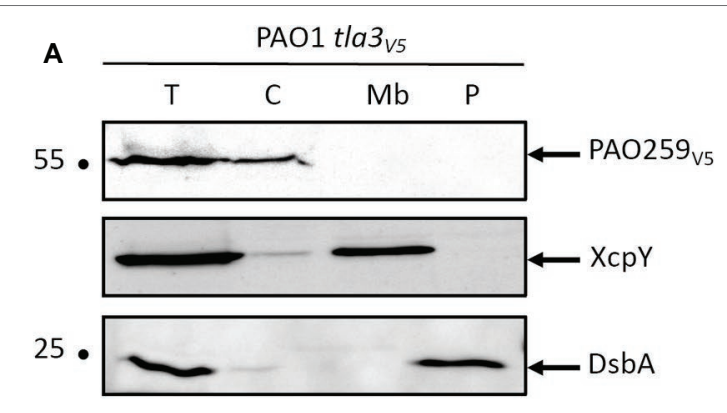

D
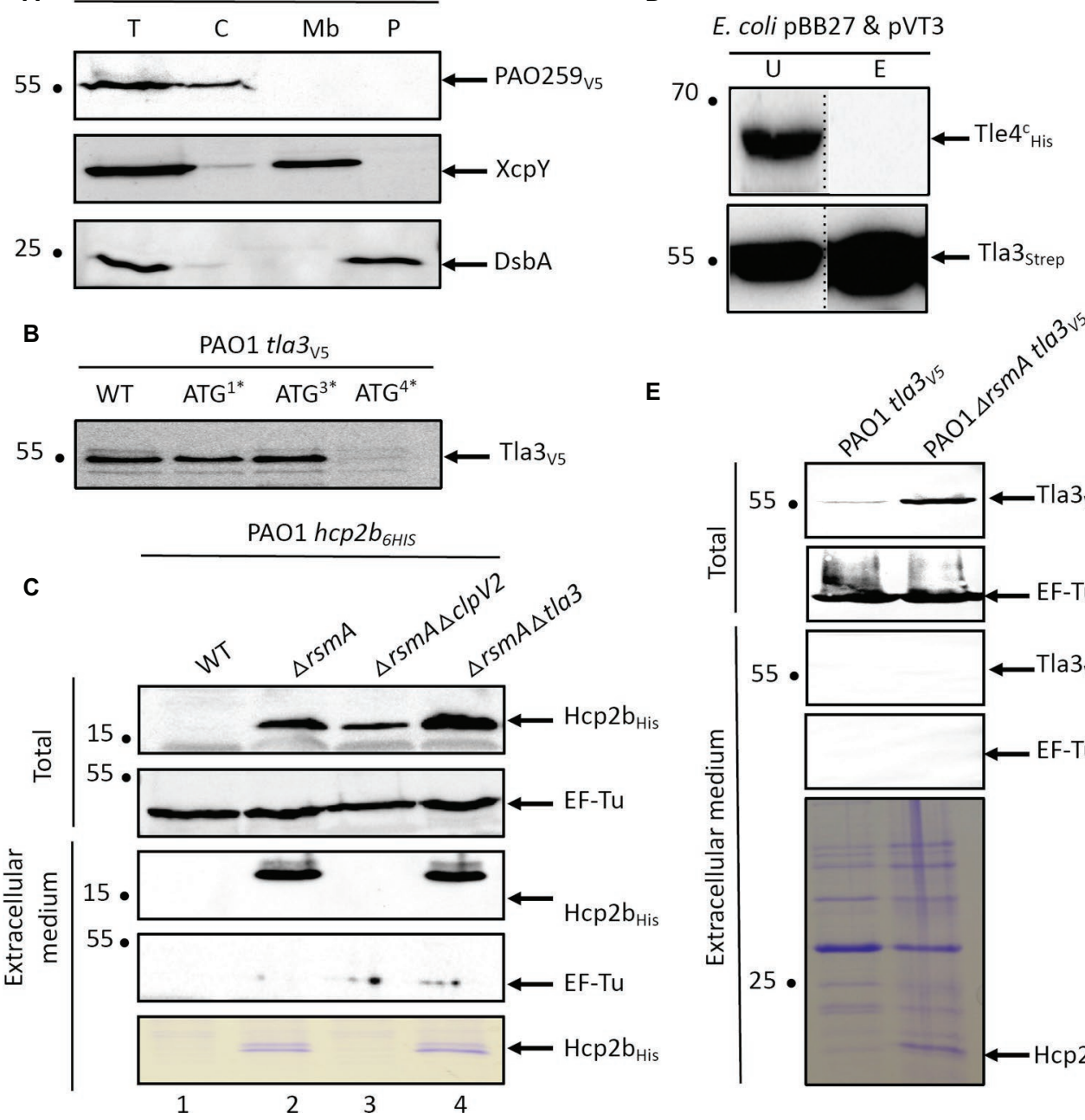

E

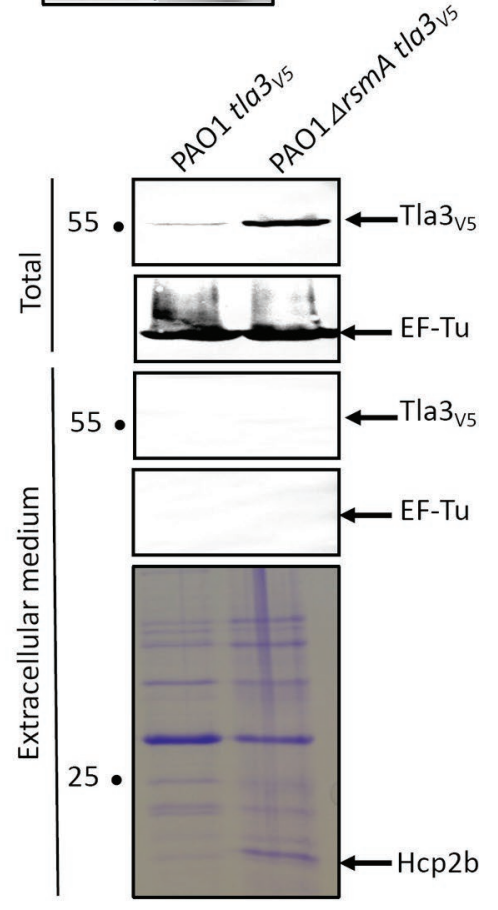

FIGURE 5 | (A) Tla3(PA0259) is a cytoplasmic protein of $P$. aeruginosa. Cells of $P$. aeruginosa PAO1 tla3 $v_{5}$ were subjected to fractionation and immunoblotting using antibodies directed against the V5 tag, XcpY and DsbA. XcpY and DsbA were used as membrane and periplasmic controls, respectively. T, whole cell; C, cytoplasm; Mb, total membrane; P, periplasm. (B) The annotated ATG drives the initiation of translation of tla3 in P. aeruginosa. Immunodetection of Tla3 ${ }_{55}$ with anti-V5 antibodies produced in a WT background or in strains in which one of the four predicted ATG in tla3 have been substituted in ATA. The number followed by a star indicates which ATG from Supplementary Figure S1 ATG 4 corresponds to the annotated ATG. (C) Tla3 is not required for Hcp2b secretion. Immunodetection of $\mathrm{Hcp}_{2} \mathrm{~b}_{6 \mathrm{His}}$ with anti-His antibodies produced in a WT background (line 1) or in strains deleted for rsmA (lines 2-4) and clpV2 (line 3) or tla3 (line 4). The strains were grown at $25^{\circ} \mathrm{C}$ for $24 \mathrm{~h}$ and total bacteria were separated from extracellular medium. Anti-EF-Tu is used as a lysis control. The extracellular

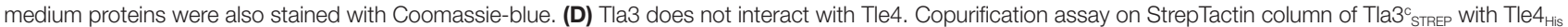
produced in E. coli BL21(DE3)pLysS from pBB27 and pVT3 respectively. Legend is as in Figure 4. (E) Tla3 is not secreted. Immunodetection of Tla3v5 with anti-HV5 antibodies produced in a WT background (line 1) or in strain deleted for rsmA (line 2). The strains were grown at $25^{\circ} \mathrm{C}$ for $24 \mathrm{~h}$ and total bacteria were separated from extracellular medium. Anti-EF-Tu is used as a lysis control. The extracellular medium proteins were also stained with Coomassie-blue. (A-E) The position of the proteins and the molecular mass markers (in kDa) are indicated.

24 hours at $37^{\circ} \mathrm{C}$ on plate with various attackers. As immunity genes are essential genes (protection from fratricide intoxication), both toxin and immunity genes have been deleted in order to construct a viable $\Delta$ tli3 $\Delta$ tle 3 mutant strain (Supplementary Table S1). Figure 6 first confirms the antibacterial function of Tle3 and the immunity role of Tli3 since the growth of the immunity mutant was affected by the WT strain because it cannot resist Tle3 toxicity, while the $\Delta$ tle3 mutant had no effect (Figure 6 compare line 1 with line 2). The use of the $\Delta c l p V 2$ strain, a H2-T6SS mutant, and of $\Delta v g r G 2 b$ and $\Delta$ tla3 mutants allowed to demonstrate that Tle3 is delivered to prey bacteria through the H2-T6SS machinery and confirms that VgrG2b and Tla3 participate in Tle3 targeting to the H2-T6SS machinery (Figure 6 compare line 1 with lines 4,5 , and 6). Indeed $\Delta c l p V 2$, $\Delta v g r G 2 b$ and $\Delta$ tla3 mutants had no effect on the immunity mutant growth since they cannot deliver Tle3 into prey bacteria. The complementation in cis of tle 3 and tla3 deletions (the mutants have been constructed for this study) restored a WT phenotype (Figure 6 compare line 2 with line 3, and line 6 with line 7) this demonstrating no polar effect on downstream 


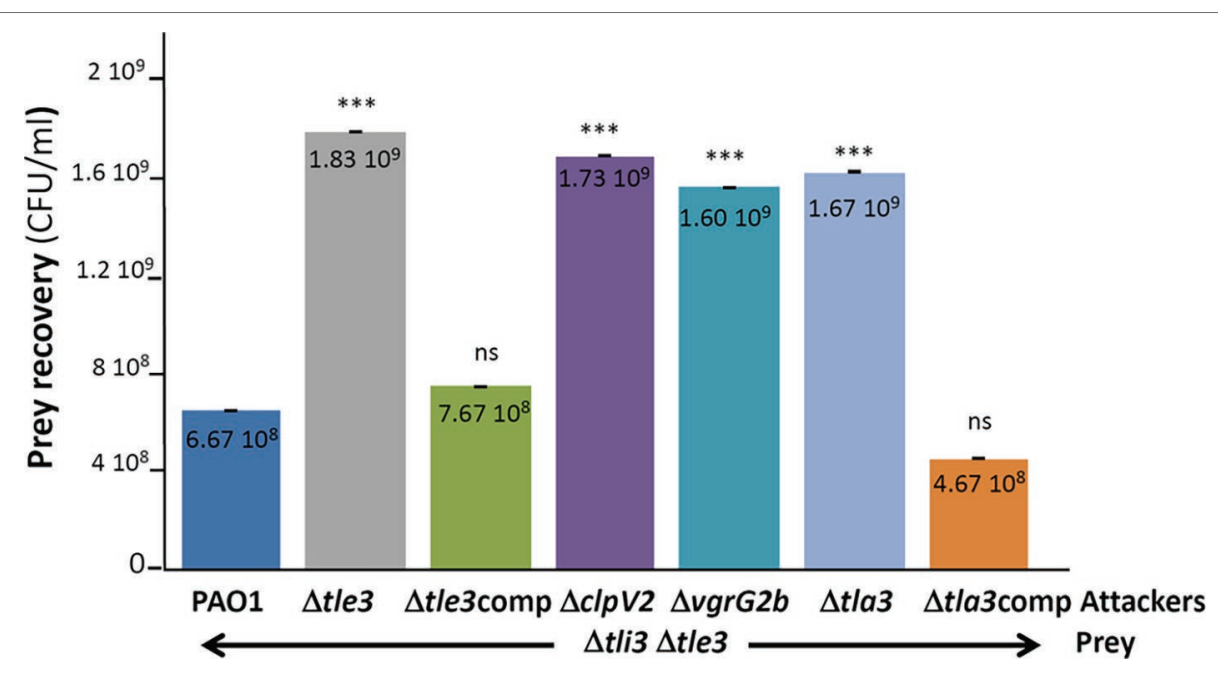

FIGURE 6 | $P$. aeruginosa growth competition. The $P$. aeruginosa prey strain ( $\Delta$ tli3 $\Delta$ tle3) was incubated with various $P$. aeruginosa attacker strains as indicated in the figure for $24 \mathrm{~h}$ at $37^{\circ} \mathrm{C}$. The number of recovered prey bacteria is indicated in CFU/ml. "comp" stand for cis complementation of the corresponding mutation with a wild-type copy inserted at the attB site on $P$. aeruginosa chromosome. Error bars represent \pm SEM $(n=3) ;{ }^{* \star \star} p<0.001$, ns not significant.

genes. Furthermore, the introduction of a wild-type copy of tli3 at the attB site on $P$. aeruginosa chromosome of the $\Delta$ tli3 $\Delta$ tle 3 mutant restores wild-type competition capacity to this strain (Supplementary Figure S4A). This confirms that the absence of the immunity was responsible of the phenotypes observed for the $\Delta$ tli3 $\Delta$ tle 3 strain (Figure 6). Finally, the $\Delta$ tla 3 mutant has been used as a prey and was not affected by the WT strain or any of the mutants excluding definitively a role of immunity as proposed by its annotation (see text footnote 1) and confirming its adaptor function (Supplementary Figure S4B).

Taken together, these results demonstrated that Tle3 is an effective H2-T6SS-dependent antibacterial toxin loaded onto the VgrG2b puncturing device via Tla3 and neutralized by Tli3 in resistant prey bacteria.

\section{VgrG2b Is a Trans-Kingdom Toxin}

A putative neutral zinc metallopeptidase domain has been predicted at the extreme C-terminus of VgrG2b by Pukatzki and colleagues (Figure 3A; Pukatzki et al., 2007). This motif (Prosite PS00142, PFAM04298) consists in a metal-binding consensus motif HExxH, the two histidine residues being ligands of the catalytic $\mathrm{Zn}^{2+}$ and the glutamic acid residue involved in nucleophilic attack. As an effector with a protease activity can target both eukaryotic and bacterial proteins, we searched for an antibacterial activity of the VgrG2b C-terminal extension. To do this, we performed the same heterologous toxicity assay in E. coli as with Tle3. As shown in Figure 7, whereas the production of $\operatorname{VgrG} 2 b_{\mathrm{Cter}}$ in the cytoplasm did not impact E. coli growth, its periplasmic production killed E. coli (Figure 7, compare lines 2 and 5). Moreover substitution of the histidine in position 935 and of the glutamic acid 936 for an alanine relieves $\operatorname{VgrG} 2 b_{\text {Cter }}$ toxicity this showing that $\operatorname{VgrG} 2 b$ is a novel antibacterial protease active in the periplasm.

Finally because of their genetic proximity, we tested whether $\mathrm{Tli} 3 \mathrm{can}$ be the immunity of $\mathrm{VgrG} 2 \mathrm{~b}_{\mathrm{Cter}}$. Indeed, the coproduction of Tli3 counteracts $\operatorname{VgrG} 2 \mathrm{~b}_{\mathrm{Cter}}$ toxicity whether it is coproduced from a second plasmid (Figure 7, line 6) or from the same transcript (Figure 7, line 7). In conclusion Tli3 serves two toxins and protects also from the antibacterial activity of VgrG2b.

\section{DISCUSSION}

Here, we report the existence of a novel pair of antibacterial effector and immunity of the H2-T6SS of $P$. aeruginosa, Tle3 (PA0260) and Tli3 (PA0261), and we propose a chronology of Tle3 secretion process that includes a cytoplasmic adaptor protein, Tla3 (PA0259) to load the toxin onto the VgrG2b spike (a model is proposed in Figure 8). Through heterologous toxicity assay and bacterial competition, we show that Tle3 was toxic once delivered in the periplasm of prey bacteria and that Tli3 can neutralize the toxin in this compartment. Interestingly, this led us to discover that VgrG2b that we previously recognized as an anti-eukaryotic effector possesses an antibacterial activity as well.

The VgrG-recruitment of cargo effectors has been previously evidenced for several antibacterial effectors among them two toxins of the Tle family, TseL (Tle2) of $V$. cholerae (Dong et al., 2013; Liang et al., 2015; Unterweger et al., 2015) and Tle1 of EAEC (Flaugnatti et al., 2016). In both cases, the tle genes were just downstream the $\operatorname{vgr} G$ genes like the organization of $\operatorname{vgrG} 2 b$ and tle3 of $P$. aeruginosa. TseL and Tle1 have been shown to directly bind a dedicated VgrG, VgrG3 and VgrG1 respectively (Dong et al., 2013; Flaugnatti et al., 2016). The domain of interaction within EAEC VgrG1 has been delimitated to the TTR domain and may also include the DUF2345, both of which are present in the $P$. aeruginosa VgrG2b. In line with this, and despite Tle3 requiring an adaptor to be targeted to VgrG2b, we have shown that Tla3 interacts with the TTR domain of VgrG2b. Taken together these data demonstrate that TTR domains of VgrGs are involved in recruitment 


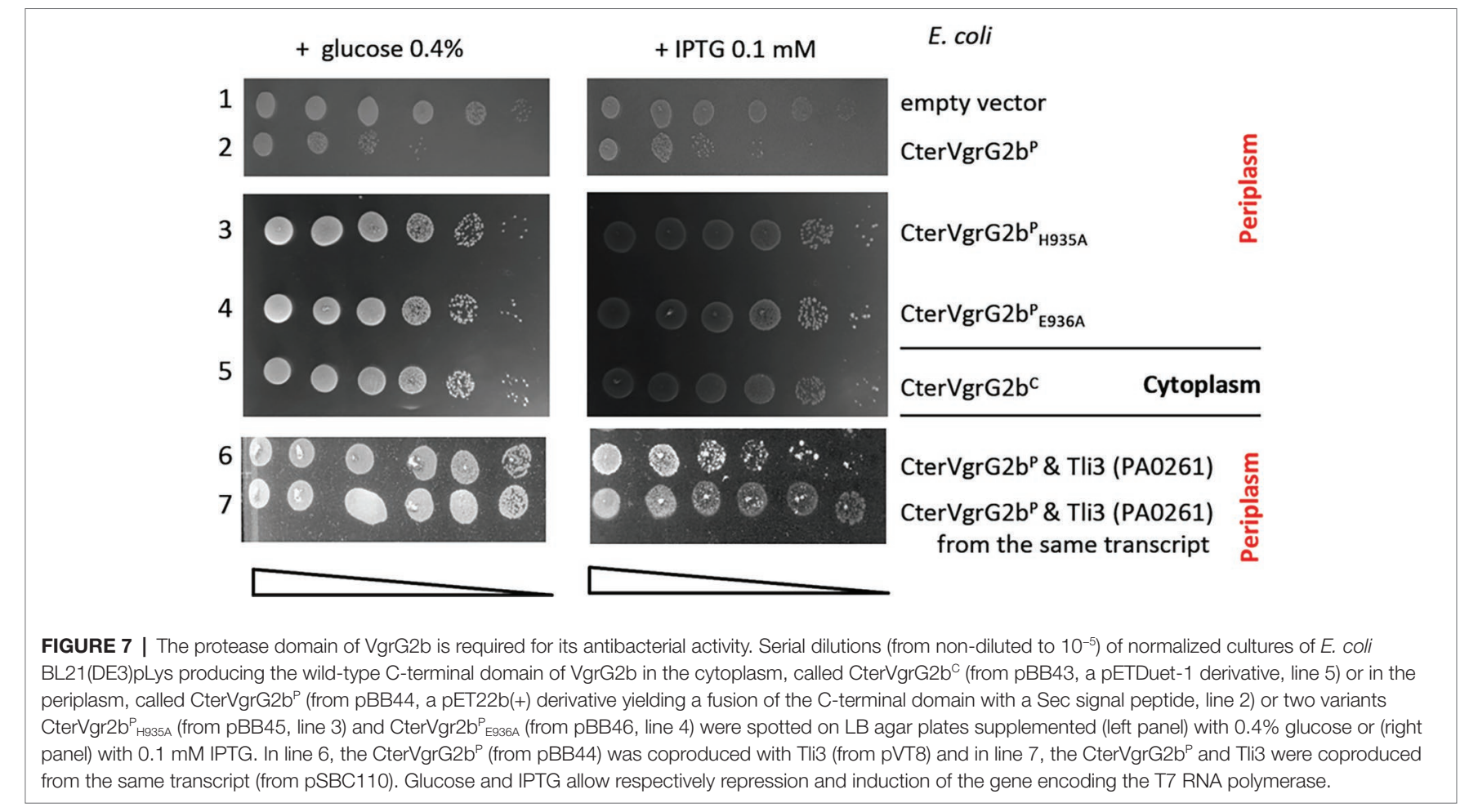

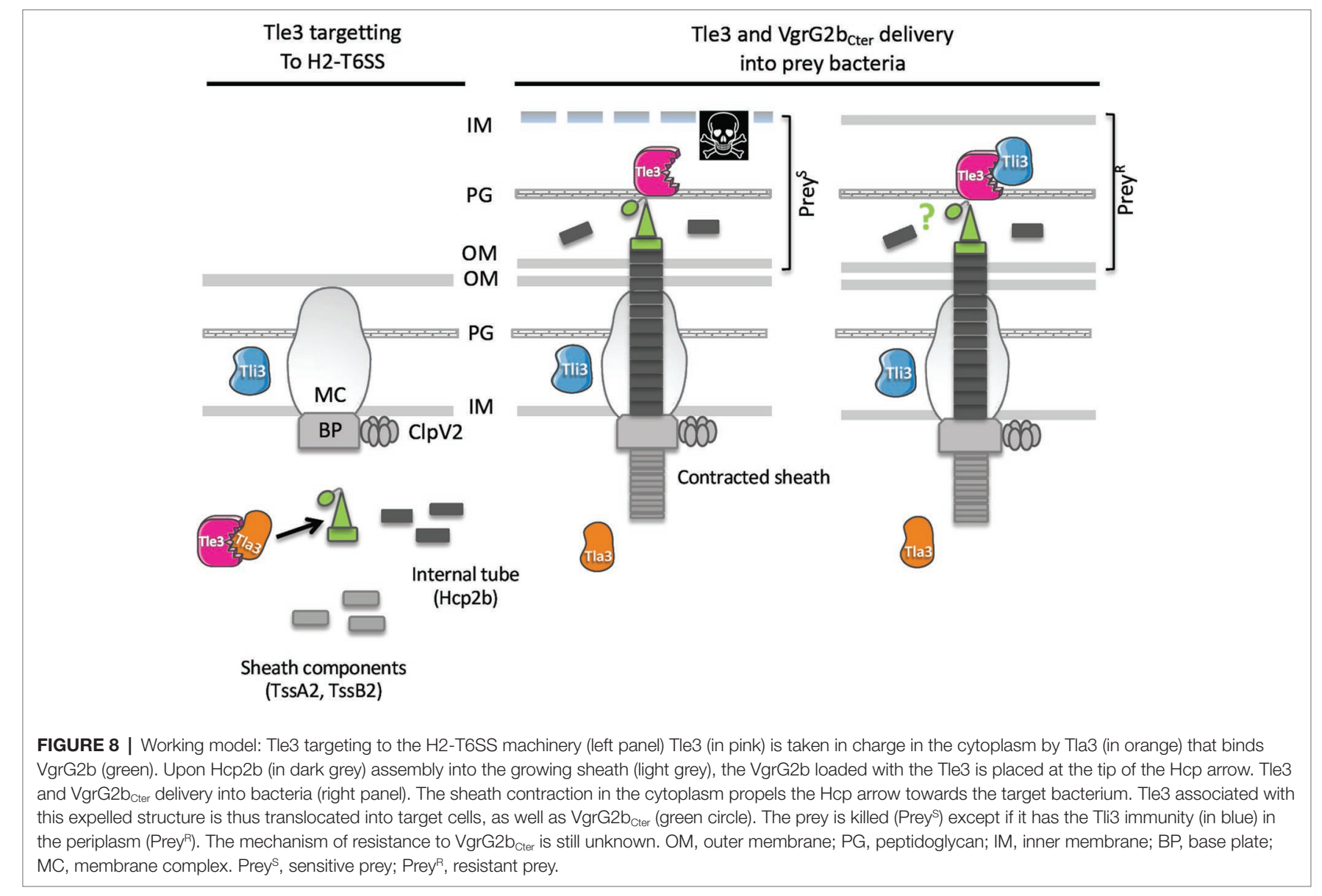


and transport of Tle effectors, directly or through adaptor. Likewise, C-terminal extensions of VgrG1 and VgrG2 of Agrobacterium tumefaciens were identified as specifically required for the delivery of each cognate DNAse toxins, named Tde1 and Tde2, respectively (Bondage et al., 2016). C-terminal domains of VgrGs can thus be considered more generally as specificity determinants for T6SS effector loading and transport.

Interestingly, TseL of $V$. cholerae requires also Tap-1 (Tec) as an adaptor protein to be delivered to another VgrG, called VgrG1 (Liang et al., 2015; Unterweger et al., 2015), showing that a sole toxin can be targeted directly and indirectly to two different VgrG proteins. Tap-1 (Tec) belongs to the DUF4123 family of adaptor proteins that contains also VasW of $V$. cholerae (Miyata et al., 2013) and several uncharacterized gene products linked to effector genes with a MIX (marker for type VI effectors) motif in Proteus mirabilis or B. thailandensis for instance (Salomon et al., 2014). Interestingly TecT, a DUF4123 adaptor of $P$. aeruginosa, has been shown to require a co-adaptor, called co-TecT, to deliver the TseT effector to the PAAR4 protein (Burkinshaw et al., 2018). This is the first example of an adaptor-co-adaptor module. Taken together, these data suggest a conserved role for DUF4123 adaptors in the recruitment of a number of T6SS effectors. Remarkably Tla3 of $P$. aeruginosa does not belong to the DUF4123 adaptor family, or to that of the two other unrelated families, the DUF1795 adaptor family, reported with EagR (effector-associated gene with Rhs) in Serratia marcescens (Alcoforado Diniz and Coulthurst, 2015) or EagT6 in P. aeruginosa (Whitney et al., 2015), and the DUF2169 adaptor family reported with Atu3641 in A. tumefaciens (Bondage et al., 2016). Nor Tla3 is a PAAR protein, the last class of effector targeting mode to a VgrG (Shneider et al., 2013; Whitney et al., 2014; Bondage et al., 2016; Cianfanelli et al., 2016a; Burkinshaw et al., 2018). Instead, we find that Tla3 harbors two DUF2875 domains (Figure 3A) that are both required for the interaction with the toxin. Moreover, genes coding DUF2875 containing proteins can be find at the vicinity of tle, tli, $\operatorname{vgrG}$, or PAAR genes, but they are restricted to $\alpha$ and $\beta$ proteobacteria (Supplementary Figure S5). We thus hypothesize that DUF2875 might assist in T6SS-mediated effector delivery. Like the three other adaptor families (DUF4123, DUF1795, and DUF2169), we have observed that Tla3 is not required for the H2-T6SS functionality since the $\Delta$ tla 3 mutant still secrete Hcp2b and can compete with a WT strain, and that Tla3 is specific for the Tle3 toxin since it did not interact with another H2-T6SS effector, TplE (Tle4). Finally, as we did not detect Tla3 secretion under constitutive H2-T6SS condition, we propose that Tla3 hands over Tle3 to VgrG2b in the cytoplasm prior to its loading to the baseplate and further recruitment to the central Hcp tube in preparation (Figure 8). Overall, the existence of various modes of effector recruitment, further refined with adaptors, likely explains how the T6SS is able to deliver numerous and structurally diverse proteins.

Five families of Tle, Tle1-5, have been identified among Gram-negative bacteria (Russell et al., 2013) and four Tle have been studied in $P$. aeruginosa so far. Our demonstration of the activity of Tle 3 in the periplasm is consistent with the observations that the heterologous periplasmic production of PldA (Tle5a) (Russell et al., 2013; Jiang et al., 2014), PldB (Tle5b)
(Jiang et al., 2014), Tle1 (Hu et al., 2014), and TplE (Tle4) (Jiang et al., 2016) is toxic. The reason of the periplasmic activity of Tle proteins is still unclear although several hypothesis have been proposed (Flaugnatti et al., 2016), the most likely being an activation of the toxin in this compartment. Very recently this has nicely been exemplified with the hijacking of DsbA in the target cells of $S$. marcescens for the activation of incoming effectors (Mariano et al., 2018). No member of the Tle3 family has yet been enzymatically characterized. Our attempts to efficiently purify Tle3 from $E$. coli or from $P$. aeruginosa have been unsuccessful, even if we have noticed that the presence of the Tla3 adaptor stabilized Tle3, it still formed inclusion bodies. In the future, we will decipher the enzymatic activity of Tle3, which is presumably active on membrane phospholipids as our preliminary data of thin-layer chromatography tend to show.

The periplasmic activity of Tle toxins is counteracted by the synthesis of a cognate immunity protein that is usually a periplasmic soluble protein, as we showed for Tli3 in P. aeruginosa, or a membrane-anchored lipoprotein (Russell et al., 2013). Interestingly the genetic organization of the tli3 gene upstream of the tle3 gene observed in E. coli, K. pneumoniae, B. cenocepacia, or R. solanacearum (Russell et al., 2013) is conserved in $P$. aeruginosa. The fact that the two genes are co-transcribed (the immunity being the first) is key for the protection against toxicity. Indeed, we have observed systematic protection against the periplasmic toxicity of Tle3 in E. coli when the two genes were expressed from the same promoter under the same plasmid, whereas it was not as efficient when the genes were on two plasmids. This genetic link reinforces the connection within the toxin/immunity pair. This has previously been noticed with a T7SS antibacterial toxin and its immunity in Staphylococcus aureus (Cao et al., 2016). Other immunities of Tle characterized so far have been shown to inhibit the action of the effector by direct protein-protein contacts (Russell et al., 2013; Jiang et al., 2014; Flaugnatti et al., 2016). Our copurification assay in E. coli demonstrates a direct interaction between Tle3 and Tli3 that was already suggested with the release of Tle3 toxicity upon coproduction of Tli3 in the periplasm. A crystal structure of the $P$. aeruginosa TplE (Tle4) effector in complex with its immunity protein TplEI (Tli4) revealed that the immunity uses a grasp mechanism to prevent the interfacial activation of the toxin ( $\mathrm{Lu}$ et al., 2014).

Like two other H2-T6SS-related orphan $\operatorname{vgrG}$ loci, the $\operatorname{vgrG} 4 b$ cluster encoding PldA (Tle5a) and the vgrG2a encoding TplE (Tle4), we show here that the $\operatorname{vgrG} 2 b$ cluster has both antibacterial activities (through Tle3 and VgrG2b) and anti-eukaryotic (through VgrG2b; Sana et al., 2015). Interestingly, VgrG2b is thus (1) a structural component of the H2-T6SS puncturing device since our bacterial competition showed its requirement for Tle3 delivery, (2) an anti-eukaryotic effector through an interaction with the microtubule nucleating complex (Sana et al., 2015), and (3) an antibacterial effector as suggested by our toxicity assay in $E$. coli. We have shown that two conserved residues of the putative metallopeptidase motif (a histidine and a glutamic acid) are essential for the VgrG2b antibacterial activity. This is consistent with an antibacterial protease activity for VgrG2b that will be, to our knowledge, the first case in the T6SS effector literature. 
The discovery of the VgrG2b immunity is even more exciting since we observed that Tli3 also relieves $\operatorname{VgrG} 2 \mathrm{~b}_{\mathrm{Cter}}$ toxicity. To our knowledge, Tli3 is the first example of a T6SS immunity serving two different proteins. But one can cite the chaperones of the type III secretion translocators that form a pore in the eukaryotic target cell membrane (Neyt and Cornelis, 1999). Indeed the chaperone SycD from Yersinia enterocolitica and its homologues from other Gram-negative bacteria bind the two translocators to prevent their premature interaction in the inner membrane and avoid further toxicity.

\section{DATA AVAILABILITY}

All datasets generated for this study are included in the manuscript and/or the Supplementary Files.

\section{AUTHOR CONTRIBUTIONS}

$\mathrm{BB}$ and $\mathrm{SB}$ designed and conceived the experiments. BB, CS, and $\mathrm{SD}$ performed the experiments. SB supervised the execution of the experiments. BB, CS, BI, and SB analyzed and discussed the data. SB wrote the paper with contribution from $\mathrm{BB}$ and reading from $\mathrm{BI}$.

\section{REFERENCES}

Alcoforado Diniz, J., and Coulthurst, S. J. (2015). Intraspecies competition in Serratia marcescens is mediated by type VI-secreted Rhs effectors and a conserved effector-associated accessory protein. J. Bacteriol. 197, 2350-2360. doi: 10.1128/JB.00199-15

Allsopp, L. P., Wood, T. E., Howard, S. A., Maggiorelli, F., Nolan, L. M., Wettstadt, S., et al. (2017). RsmA and AmrZ orchestrate the assembly of all three type VI secretion systems in Pseudomonas aeruginosa. Proc. Natl. Acad. Sci. USA 114, 7707-7712. doi: 10.1073/pnas.1700286114

Basler, M. (2015). Type VI secretion system: secretion by a contractile nanomachine. Philos. Trans. R. Soc. Lond. Ser. B Biol. Sci. 370. doi: 10.1098/rstb.2015.0021

Battesti, A., and Bouveret, E. (2012). The bacterial two-hybrid system based on adenylate cyclase reconstitution in Escherichia coli. Methods 58, 325-334. doi: 10.1016/j.ymeth.2012.07.018

Benz, J., and Meinhart, A. (2014). Antibacterial effector/immunity systems: it's just the tip of the iceberg. Curr. Opin. Microbiol. 17, 1-10. doi: 10.1016/j. mib.2013.11.002

Bleves, S. (2016). Game of trans-kingdom effectors. Trends Microbiol. 24, 773-774. doi: 10.1016/j.tim.2016.08.002

Bleves, S., Viarre, V., Salacha, R., Michel, G. P. F., Filloux, A., and Voulhoux, R. (2010). Protein secretion systems in Pseudomonas aeruginosa: a wealth of pathogenic weapons. Int. J. Med. Microbiol. 300, 534-543. doi: 10.1016/j. ijmm.2010.08.005

Bondage, D. D., Lin, J.-S., Ma, L.-S., Kuo, C.-H., and Lai, E.-M. (2016). VgrG $\mathrm{C}$ terminus confers the type VI effector transport specificity and is required for binding with PAAR and adaptor-effector complex. Proc. Natl. Acad. Sci. USA 113, E3931-E3940. doi: 10.1073/pnas.1600428113

Burkinshaw, B. J., Liang, X., Wong, M., Le, A. N. H., Lam, L., and Dong, T. G. (2018). A type VI secretion system effector delivery mechanism dependent on PAAR and a chaperone-co-chaperone complex. Nat. Microbiol. 3, 632-640. doi: 10.1038/s41564-018-0144-4

Cao, Z., Casabona, M. G., Kneuper, H., Chalmers, J. D., and Palmer, T. (2016). The type VII secretion system of Staphylococcus aureus secretes a nuclease toxin that targets competitor bacteria. Nat. Microbiol. 2:16183. doi: 10.1038/ nmicrobiol.2016.183

\section{FUNDING}

This work was supported by recurrent funding from the CNRS and Aix-Marseille University. The project leading to this publication has received funding from the Excellence Initiative of Aix-Marseille University-A*Midex, a French "Investissements d'Avenir" program ("Emergence \& Innovation" A-M-AAP-EI-17139-170301-10.31-BLEVES-HLS).

\section{ACKNOWLEDGMENTS}

We thank V. Tutagata for pVT1, pVT8, and pVT9 constructs, and $\mathrm{B}$. Douzi for all the advices during protein purification and members of B.B. $\mathrm{PhD}$ committee for helpful discussion and support. We are grateful to M. Ba, I. Bringer, A. Brun, and O. Uderso for technical assistance. $\mathrm{BB}$ was financed with a $\mathrm{PhD}$ fellowship from the French Research Ministry.

\section{SUPPLEMENTARY MATERIAL}

The Supplementary Material for this article can be found online at: https://www.frontiersin.org/articles/10.3389/fmicb.2019.01218/ full\#supplementary-material

Cianfanelli, F. R., Alcoforado Diniz, J., Guo, M., De Cesare, V., Trost, M., and Coulthurst, S. J. (2016a). VgrG and PAAR proteins define distinct versions of a functional type VI secretion system. PLoS Pathog. 12:e1005735. doi: 10.1371/journal.ppat.1005735

Cianfanelli, F. R., Monlezun, L., and Coulthurst, S. J. (2016b). Aim, load, fire: the type VI secretion system, a bacterial nanoweapon. Trends Microbiol. 24, 51-62. doi: 10.1016/j.tim.2015.10.005

Dong, T. G., Ho, B. T., Yoder-Himes, D. R., and Mekalanos, J. J. (2013). Identification of T6SS-dependent effector and immunity proteins by Tn-seq in Vibrio cholerae. Proc. Natl. Acad. Sci. USA 110, 2623-2628. doi: 10.1073/ pnas. 1222783110

Flaugnatti, N., Le, T. T. H., Canaan, S., Aschtgen, M.-S., Nguyen, V. S., Blangy, S., et al. (2016). A phospholipase A1 antibacterial Type VI secretion effector interacts directly with the C-terminal domain of the VgrG spike protein for delivery. Mol. Microbiol. 99, 1099-1118. doi: 10.1111/mmi.13292

Hecht, A. L., Casterline, B. W., Earley, Z. M., Goo, Y. A., Goodlett, D. R., and Bubeck Wardenburg, J. (2016). Strain competition restricts colonization of an enteric pathogen and prevents colitis. EMBO Rep. 17, 1281-1291. doi: $10.15252 / \mathrm{embr} .201642282$

Hood, R. D., Singh, P., Hsu, F., Güvener, T., Carl, M. A., Trinidad, R. R. S., et al. (2010). A type VI secretion system of Pseudomonas aeruginosa targets a toxin to bacteria. Cell Host Microbe 7, 25-37. doi: 10.1016/j.chom.2009.12.007

Hu, H., Zhang, H., Gao, Z., Wang, D., Liu, G., Xu, J., et al. (2014). Structure of the type VI secretion phospholipase effector Tlel provides insight into its hydrolysis and membrane targeting. Acta Crystallogr. D Biol. Crystallogr. 70, 2175-2185. doi: 10.1107/S1399004714012899

Ize, B., Viarre, V., and Voulhoux, R. (2014). Cell fractionation. Methods Mol. Biol. 1149, 185-191. doi: 10.1007/978-1-4939-0473-0_15

Jeong, J.-Y., Yim, H.-S., Ryu, J.-Y., Lee, H. S., Lee, J.-H., Seen, D.-S., et al. (2012). One-step sequence- and ligation-independent cloning as a rapid and versatile cloning method for functional genomics studies. Appl. Environ. Microbiol. 78, 5440-5443. doi: 10.1128/AEM.00844-12

Jiang, F., Wang, X., Wang, B., Chen, L., Zhao, Z., Waterfield, N. R., et al. (2016). The Pseudomonas aeruginosa type VI secretion PGAP1-like effector induces host autophagy by activating endoplasmic reticulum stress. Cell Rep. 16, 1502-1509. doi: 10.1016/j.celrep.2016.07.012 
Jiang, F., Waterfield, N. R., Yang, J., Yang, G., and Jin, Q. (2014). A Pseudomonas aeruginosa type VI secretion phospholipase D effector targets both prokaryotic and eukaryotic cells. Cell Host Microbe 15, 600-610. doi: 10.1016/j. chom.2014.04.010

Karimova, G., Pidoux, J., Ullmann, A., and Ladant, D. (1998). A bacterial two-hybrid system based on a reconstituted signal transduction pathway. Proc. Natl. Acad. Sci. USA 95, 5752-5756.

Kolaskar, A. S., and Reddy, B. V. (1985). A method to locate protein coding sequences in DNA of prokaryotic systems. Nucleic Acids Res. 13, 185-194. doi: 10.1093/nar/13.1.185

LaCourse, K. D., Peterson, S. B., Kulasekara, H. D., Radey, M. C., Kim, J., and Mougous, J. D. (2018). Conditional toxicity and synergy drive diversity among antibacterial effectors. Nat. Microbiol. 3, 440-446. doi: 10.1038/ s41564-018-0113-y

Liang, X., Moore, R., Wilton, M., Wong, M. J. Q., Lam, L., and Dong, T. G. (2015). Identification of divergent type VI secretion effectors using a conserved chaperone domain. Proc. Natl. Acad. Sci. USA 112, 9106-9111. doi: 10.1073/ pnas. 1505317112

Lu, D., Zheng, Y., Liao, N., Wei, L., Xu, B., Liu, X., et al. (2014). The structural basis of the Tle4-Tli4 complex reveals the self-protection mechanism of H2-T6SS in Pseudomonas aeruginosa. Acta Crystallogr. D Biol. Crystallogr. 70, 3233-3243. doi: 10.1107/S1399004714023967

MacIntyre, D. L., Miyata, S. T., Kitaoka, M., and Pukatzki, S. (2010). The Vibrio cholerae type VI secretion system displays antimicrobial properties. Proc. Natl. Acad. Sci. USA 107, 19520-19524. doi: 10.1073/pnas.1012931107

Mariano, G., Monlezun, L., and Coulthurst, S. J. (2018). Dual role for DsbA in attacking and targeted bacterial cells during type VI secretion systemmediated competition. Cell Rep. 22, 774-785. doi: 10.1016/j.celrep.2017.12.075

Miyata, S. T., Unterweger, D., Rudko, S. P., and Pukatzki, S. (2013). Dual expression profile of type VI secretion system immunity genes protects pandemic Vibrio cholerae. PLoS Pathog. 9:e1003752. doi: 10.1371/journal. ppat. 1003752

Mougous, J. D., Cuff, M. E., Raunser, S., Shen, A., Zhou, M., Gifford, C. A., et al. (2006). A virulence locus of Pseudomonas aeruginosa encodes a protein secretion apparatus. Science 312, 1526-1530. doi: 10.1126/ science. 1128393

Neyt, C., and Cornelis, G. R. (1999). Role of SycD, the chaperone of the Yersinia Yop translocators YopB and YopD. Mol. Microbiol. 31, 143-156. doi: 10.1046/j.1365-2958.1999.01154.x

Nielsen, H. (2017). "Predicting secretory proteins with SignalP" in Protein function prediction. ed. D. Kihara (New York, NY: Springer), 59-73.

Pukatzki, S., Ma, A. T., Revel, A. T., Sturtevant, D., and Mekalanos, J. J. (2007). Type VI secretion system translocates a phage tail spike-like protein into target cells where it cross-links actin. Proc. Natl. Acad. Sci. USA 104, 15508-15513. doi: 10.1073/pnas.0706532104

Pukatzki, S., Ma, A. T., Sturtevant, D., Krastins, B., Sarracino, D., Nelson, W. C., et al. (2006). Identification of a conserved bacterial protein secretion system in Vibrio cholerae using the Dictyostelium host model system. Proc. Natl. Acad. Sci. USA 103, 1528-1533. doi: 10.1073/pnas.0510322103

Russell, A. B., LeRoux, M., Hathazi, K., Agnello, D. M., Ishikawa, T., Wiggins, P. A., et al. (2013). Diverse type VI secretion phospholipases are functionally plastic antibacterial effectors. Nature 496, 508-512. doi: 10.1038/ nature 12074

Salomon, D., Kinch, L. N., Trudgian, D. C., Guo, X., Klimko, J. A., Grishin, N. V., et al. (2014). Marker for type VI secretion system effectors. Proc. Natl. Acad. Sci. USA 111, 9271-9276. doi: 10.1073/pnas.1406110111

Sana, T. G., Baumann, C., Merdes, A., Soscia, C., Rattei, T., Hachani, A., et al. (2015). Internalization of Pseudomonas aeruginosa strain PAO1 into epithelial cells is promoted by interaction of a T6SS effector with the microtubule network. MBio 6:e00712. doi: 10.1128/mBio.00712-15

Sana, T. G., Berni, B., and Bleves, S. (2016a). The T6SSs of Pseudomonas aeruginosa strain PAO1 and their effectors: beyond bacterial-cell targeting. Front. Cell. Infect. Microbiol. 6:61. doi: 10.3389/fcimb.2016.00061

Sana, T. G., Flaugnatti, N., Lugo, K. A., Lam, L. H., Jacobson, A., Baylot, V. et al. (2016b). Salmonella Typhimurium utilizes a T6SS-mediated antibacterial weapon to establish in the host gut. Proc. Natl. Acad. Sci. USA 113, E5044-E5051. doi: 10.1073/pnas.1608858113

Sana, T. G., Hachani, A., Bucior, I., Soscia, C., Garvis, S., Termine, E., et al. (2012). The second type VI secretion system of Pseudomonas aeruginosa strain PAO1 is regulated by quorum sensing and Fur and modulates internalization in epithelial cells. J. Biol. Chem. 287, 27095-27105. doi: 10.1074/jbc.M112.376368

Shneider, M. M., Buth, S. A., Ho, B. T., Basler, M., Mekalanos, J. J., and Leiman, P. G. (2013). PAAR-repeat proteins sharpen and diversify the type VI secretion system spike. Nature 500, 350-353. doi: 10.1038/nature12453

Unterweger, D., Kostiuk, B., Ötjengerdes, R., Wilton, A., Diaz-Satizabal, L., and Pukatzki, S. (2015). Chimeric adaptor proteins translocate diverse type VI secretion system effectors in Vibrio cholerae. EMBO J. 34, 2198-2210. doi: 10.15252/embj.201591163

Whitney, J. C., Beck, C. M., Goo, Y. A., Russell, A. B., Harding, B. N., De Leon, J. A., et al. (2014). Genetically distinct pathways guide effector export through the type VI secretion system. Mol. Microbiol. 92, 529-542. doi: $10.1111 / \mathrm{mmi} .12571$

Whitney, J. C., Quentin, D., Sawai, S., LeRoux, M., Harding, B. N., Ledvina, H. E., et al. (2015). An interbacterial $\operatorname{NAD}(\mathrm{P})(+)$ glycohydrolase toxin requires elongation factor Tu for delivery to target cells. Cell 163, 607-619. doi: 10.1016/j.cell.2015.09.027

WHO (2019). WHO publishes list of bacteria for which new antibiotics are urgently needed. Available at: https://www.who.int/news-room/detail/2702-2017-who-publishes-list-of-bacteria-for-which-new-antibiotics-are-urgentlyneeded (Accessed January 21, 2019).

Conflict of Interest Statement: The authors declare that the research was conducted in the absence of any commercial or financial relationships that could be construed as a potential conflict of interest.

Copyright (c) 2019 Berni, Soscia, Djermoun, Ize and Bleves. This is an open-access article distributed under the terms of the Creative Commons Attribution License (CC BY). The use, distribution or reproduction in other forums is permitted, provided the original author(s) and the copyright owner(s) are credited and that the original publication in this journal is cited, in accordance with accepted academic practice. No use, distribution or reproduction is permitted which does not comply with these terms. 